\title{
Uniform Circular Motion
}

A particle is in uniform circular motion if it travels in a circular path at a constant speed. Orbiting satellites, ceiling fans and vinyl records are a few objects that display uniform circular motion. Even though its speed remains constant, a particle in uniform circular motion is constantly accelerating. This can initially be a little counterintuitive, but it stems from the definition that acceleration is the rate of change of velocity which is a vector quantity, having both magnitude and direction. Since the direction of the speed continuously changes when a particle moves in a circular path, the velocity also continuously changes and hence there is a constant acceleration.

\section{Centripetal Acceleration}

Consider a particle moving in a circular path of radius $r$ with a constant speed $v$, as shown in Fig. 1. At a particular time $t$ let the velocity of the particle be $\vec{v}_{1}$ and after a short time $\Delta t$ let the velocity be $\vec{v}_{2}$. Here, $\Delta \theta$ is the angle swept and $s$ is the distance traveled by the particle. 


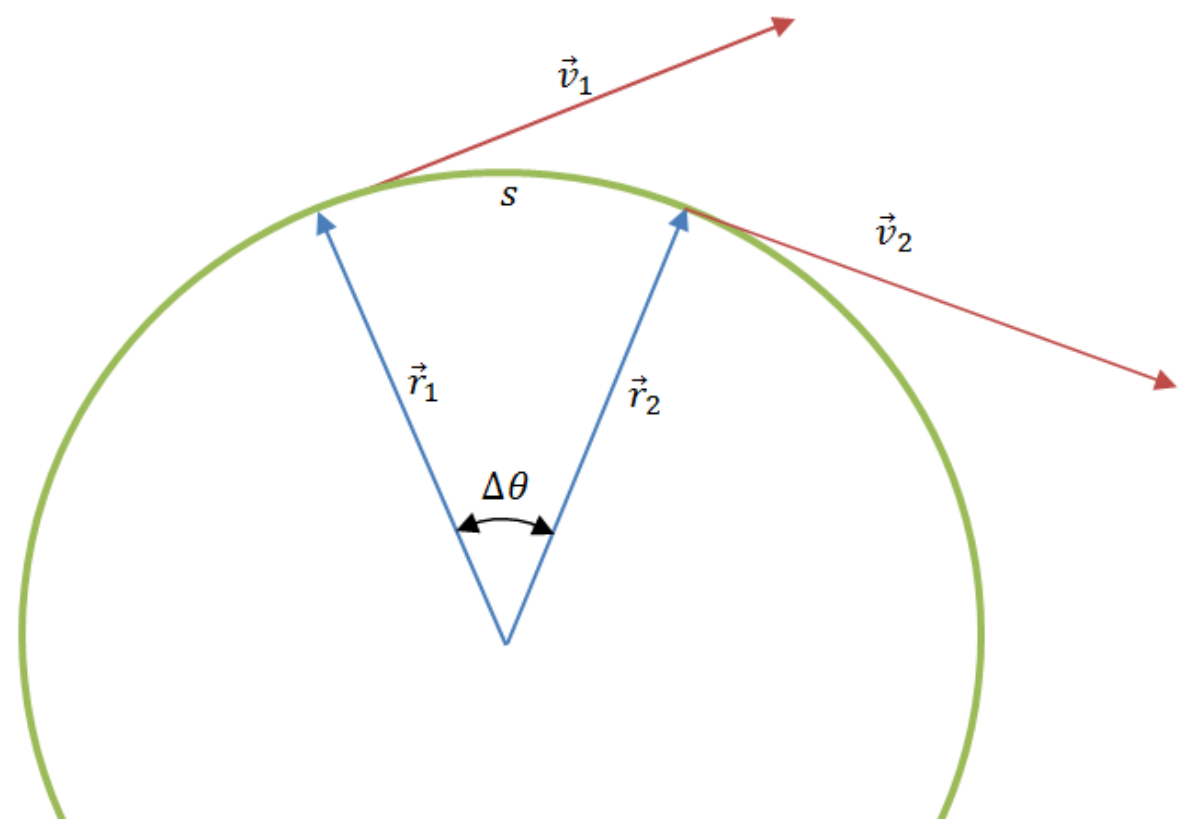

Fig. 1: Particle in uniform circular motion

The average acceleration can then be defined as

$$
\vec{a}_{a v g}=\frac{\vec{v}_{2}-\vec{v}_{1}}{\Delta t}=\frac{\Delta \vec{v}}{\Delta t}
$$

The following image shows the subtraction of the of the two velocity vectors. 


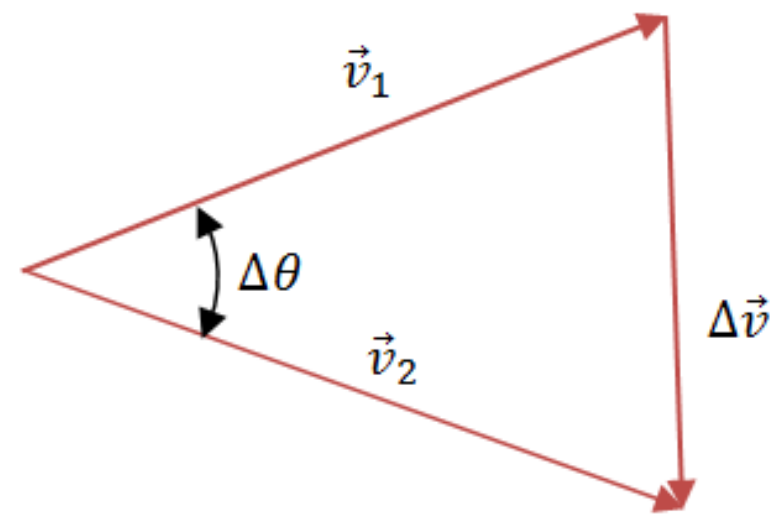

Fig. 2: Vector subtraction

If the time interval is shortened then $\Delta \vec{v}$ becomes smaller and almost perpendicular to $\vec{v}_{1}$, as shown below.

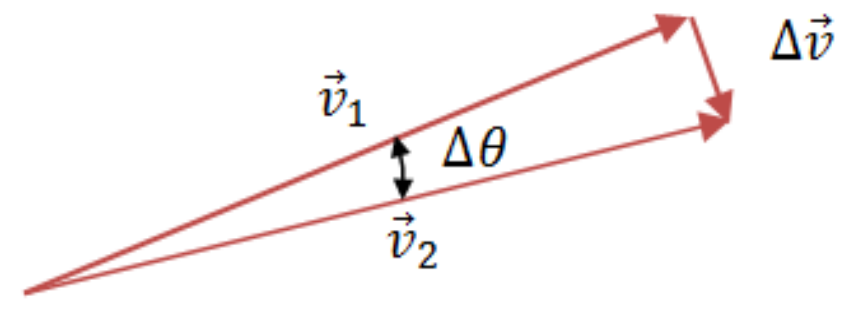

Fig. 3: Vector subtraction - small angle

For a very small angle, using geometry, we can write

$$
\Delta v \approx v_{1} \cdot \Delta \theta \approx v \cdot \Delta \theta
$$

Also, from geometry, 


$$
\Delta \theta=\frac{s}{r}=\frac{v \cdot \Delta t}{r}
$$

If we take the limit as $\Delta t \rightarrow 0$, we can write

$$
d v=v \cdot d \theta \quad \text { and } \quad d \theta=\frac{v \cdot d t}{r}
$$

Therefore, the magnitude of the acceleration, at the instant when the velocity is $\vec{v}_{1}$, is

$$
\frac{d v}{d t}=\frac{v \cdot v \cdot d t}{r \cdot d t}
$$

which simplifies to

$$
a=\frac{v^{2}}{r}
$$

The direction of this acceleration can be obtained from Fig. 3. As $\Delta \theta \rightarrow 0, \Delta \vec{v}$ becomes perpendicular to $\overrightarrow{v_{1}}$ and points radially inward to the center of the circle. This acceleration is called the centripetal acceleration.

\section{Example 1: Centripetal Acceleration}

Problem Statement: A disk with a radius of $0.1 \mathrm{~m}$ is spinning about its central axis at a uniform rate. The velocity of a point on the edge of the disk are $1 \mathrm{~m} / \mathrm{s}$. What is the centripetal acceleration of a point on this disk located $0.05 \mathrm{~m}$ from the axis of rotation.

restart;

Data:

$v_{l}:=1: \quad[\mathrm{m} / \mathrm{s}]$

$r_{1}:=0.1: \quad[\mathrm{m}]$ 


$$
\begin{gathered}
r_{2} \quad{ }^{:=0.1} \\
05:
\end{gathered}
$$

\section{Solution:}

Since the two points are located at different distances from the axis of rotation, they cover different distances per unit time. However, the angle swept per unit time stays the same. The angle swept by the outer point is

$\theta=\frac{v_{1} \cdot t}{r_{1}}$

and the angle swept by the inner point is

$\theta=\frac{v_{2} \cdot t}{r_{2}}$

Equating these two angles, yields

$v_{2}:=\frac{v_{1} \cdot r_{2}}{r_{1}}:$

And the centripetal acceleration using Eq. (2) is,

$a:=\frac{\left(v_{2}\right)^{2}}{r_{2}}=5.00$

Therefore a point located $0.05 \mathrm{~m}$ from the central axis experiences a centripetal acceleration of $5 \mathrm{~m} / \mathrm{s}^{2}$. The following is a plot of the centripetal acceleration vs. radius for points on the disk. This plot shows that the centripetal acceleration increases linearly with the radius when all the points are rotating together with the same rate of angular 
motion.

Plot for Centripetal Acceleration vs. Radius

Centripetal Acceleration vs. Radius

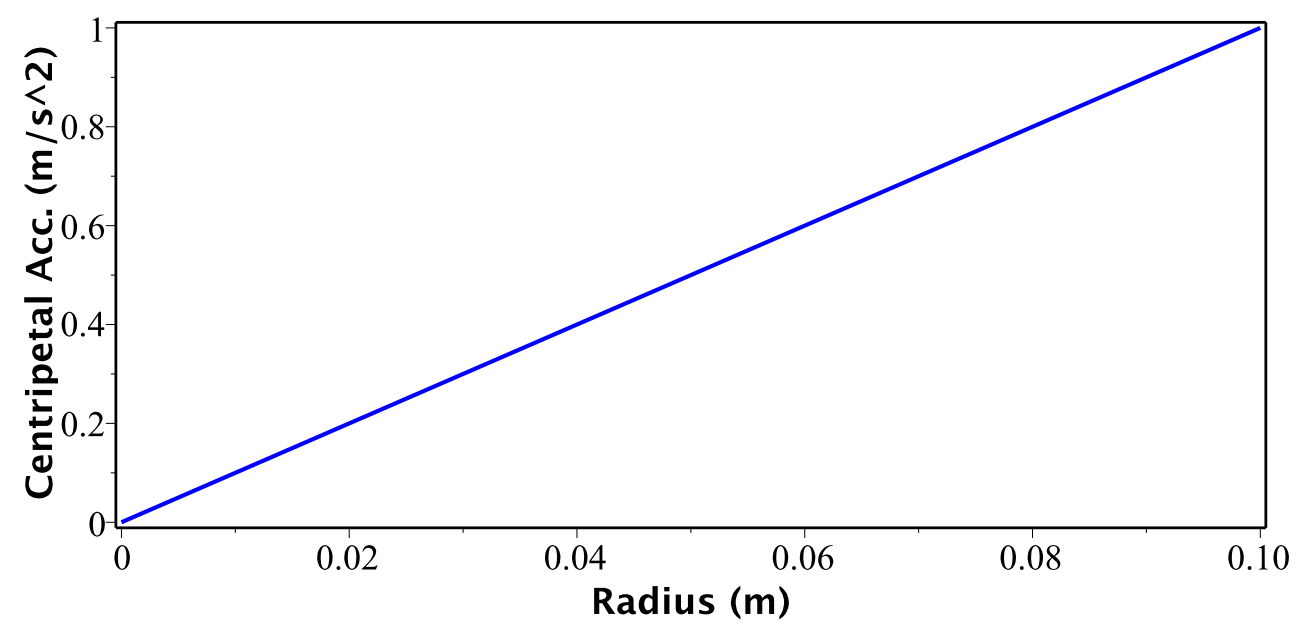

\section{Time Period and Frequency}

The time period $T$ is defined as the time taken by an object in uniform circular motion to complete one full circle.

$$
T=\frac{2 \cdot \pi \cdot r}{v}
$$

Here $r$ is the radius of the circular path and $v$ is the speed. Additionally, the frequency $f$ is defined as the number of full circles the object completes per unit time.

$$
f=\frac{1}{T}
$$




\section{An Example with MapleSim}

\section{Example 2: Human Centrifuge}

Problem Statement: A human centrifuge, used for training fighter pilots, is rotating at a rate of $45 \mathrm{rpm}$. The distance between the pilot and the axis of rotation is $5 \mathrm{~m}$. What is the centripetal acceleration of the pilot?

\section{Analytical Solution}

restart :

\section{Data:}

$n:=45: \quad[\mathrm{rpm}]$

$r:=5: \quad[\mathrm{m}]$

$g^{:=9.1^{\left[\mathrm{m} / \mathrm{s}^{2}\right]}}$

$81:$

\section{Solution:}

Since the rate of rotation is $45 \mathrm{rpm}$, the speed of the pilot is

$v:=\frac{n \cdot 2 \cdot \mathrm{Pi} \cdot r}{60}$

$$
\frac{15}{2} \pi
$$

The magnitude of the centripetal acceleration, given by Eq. (2) is

$a:=\frac{v^{2}}{r}$

$$
\frac{45}{4} \pi^{2}
$$

$\stackrel{\text { at } 5 \text { digits }}{\longrightarrow}$

$$
111.03
$$

In $g$ units, this is

$$
a_{g}:=\frac{a}{g}
$$


at 5 digits

$$
\frac{45}{4} \frac{\pi^{2}}{g}
$$

111.03

$g$

Therefore the pilot experiences approximately $11 \mathrm{~g}$ of centripetal acceleration.

\section{MapleSim Simulation}

\section{Constructing the Model}

Step 1: Insert components

Drag the following components into the workspace:

Table 1: Components and locations

\begin{tabular}{|c|c|}
\hline $\begin{array}{l}\text { Comp } \\
\text { onent }\end{array}$ & Location \\
\hline 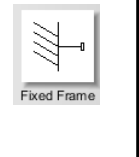 & $\begin{array}{l}\text { Multibody > } \\
\text { Body and } \\
\text { Frames }\end{array}$ \\
\hline 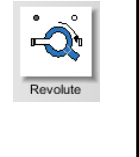 & $\begin{array}{l}\text { Multibody > } \\
\text { Joints and } \\
\text { Motions }\end{array}$ \\
\hline 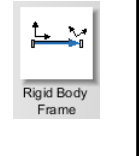 & $\begin{array}{l}\text { Multibody > } \\
\text { Body and } \\
\text { Frames }\end{array}$ \\
\hline$\stackrel{-1}{\text { Rुg }}$ & $\begin{array}{c}\text { Multibody > } \\
\text { Body and } \\
\text { Frames }\end{array}$ \\
\hline 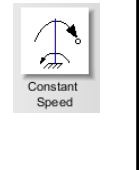 & $\begin{array}{l}\text { 1-D Mechanical } \\
>\text { Rotational > } \\
\text { Motion Drivers }\end{array}$ \\
\hline 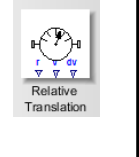 & $\begin{array}{l}\text { 1-D Mechanical } \\
>\text { Rotational > } \\
\text { Motion Drivers }\end{array}$ \\
\hline
\end{tabular}


Step 2: Connect the components

Connect the components as shown in the following diagram (the dashed boxes are not part of the model, they have been drawn on top to help make it clear what the different components are for).

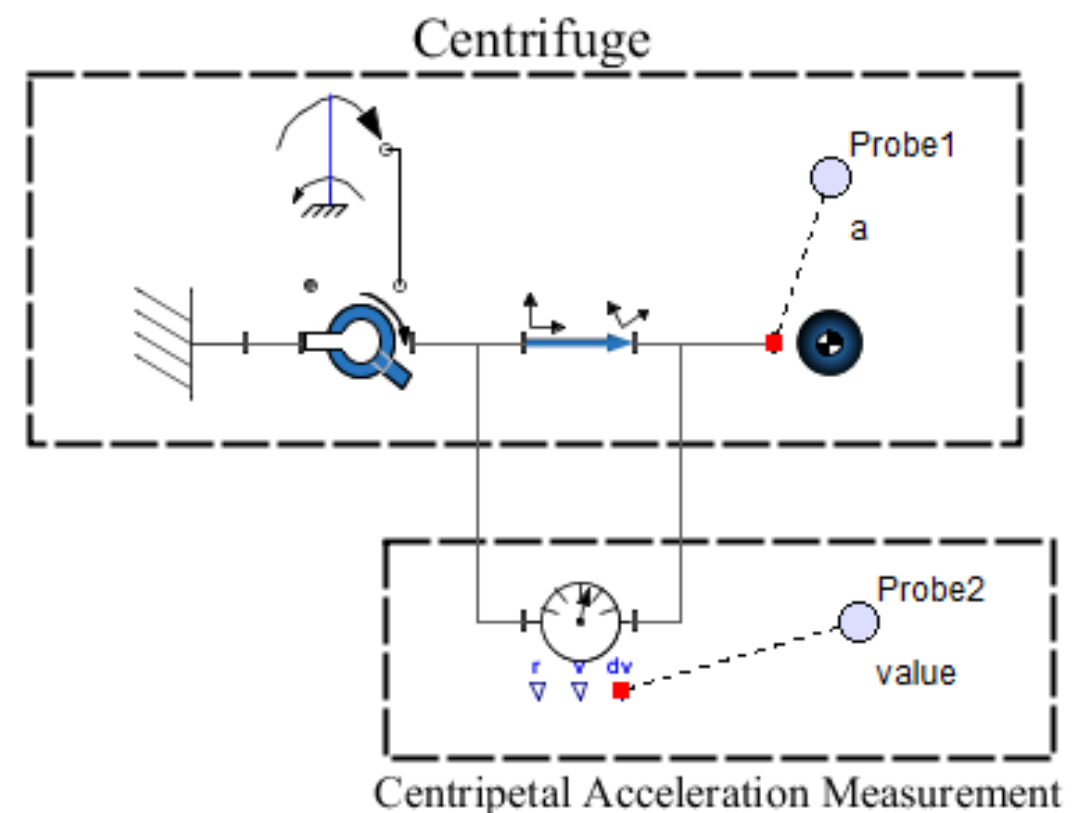

Fig. 4: Component diagram

Step 3: Create parameters

Click the Add a Parameter Block icon (馗 ), click on the workspace and double click the Parameters icon that appears on the workspace. Create parameters for the rate of rotation $n$ and the distance from the axis of rotation $r$ (as shown below).

Parameters subsystem default setting

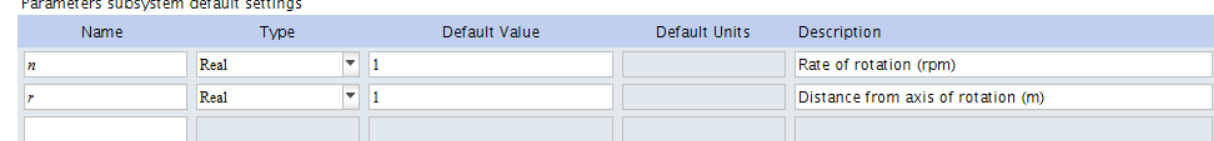

Fig. 5: Parameter Block

Step 4: Adjust the parameters 
Return to the main diagram ( $\because>$ Main $-\quad$ ) and, with a single click on the

Parameters icon, enter the following parameters (Fig. 6) in the Inspector tab.

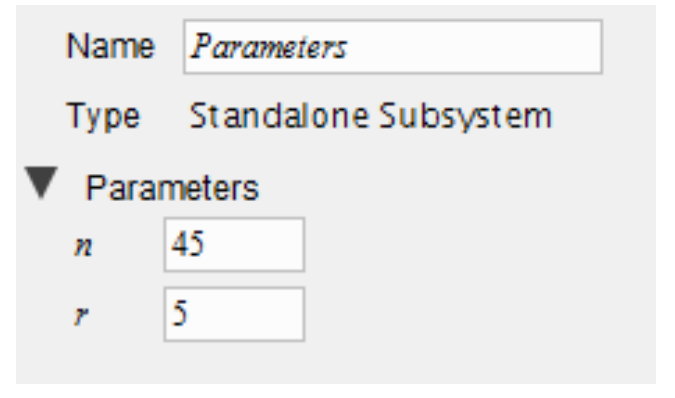

Fig. 6: Parameters

Note: Step 3 and Step 4 are not essential and can be skipped. The parameter values can be directly entered for each component instead of using variables. However, creating a parameter block as described above makes it easy to repeatedly change the parameters and play around with the model to see the effects on the simulation result.

Step 5: Set up the Centrifuge

1. Click the Revolute component and, in the Inspector tab, select $[\mathbf{0 , 1 , 0 ]}$ for the axis of rotation $\left(\widehat{e}_{1}\right)$.

2. Click the Rigid Body Frame component and enter $[r, 0,0]$ for the $x, y, z$ offset ( $\left.\bar{r}_{X Y Z}\right)$.

3. Click the Rotational Constant Speed component and enter $\boldsymbol{n}$ for the fixed speed $\left(\boldsymbol{\omega}_{\text {fixed }}\right)$. Also, change the units to rpm.

4. Click the Probe connected to the Rigid Body component and select 1, 2, and 3 under Acceleration in the Inspector tab. This probe will show the acceleration components along the $\mathrm{x}, \mathrm{y}$ and $\mathrm{z}$ axes.

Step 6: Set up the sensor for the centripetal acceleration

1. Connect the Relative Translation sensor across the Rigid Body Frame component in a parallel configuration, as shown in Fig. 4.

2. Attach a Probe to the dv port of the Relative Translation sensor. 
3. Click this Probe and select $\mathbf{1}$ in the Inspector tab. This probe will show the sensor measurement of the acceleration in the direction along the frame that connects the object to the axis of rotation. This is the centripetal acceleration.

Step 7: Run the Simulation

In the Settings tab, reduce the Simulation duration $\left(t_{d}\right)$ to $1 \mathrm{~s}$ (a long simulation time is not required). Then run the simulation.

The following image shows the 3-D view for the simulation.

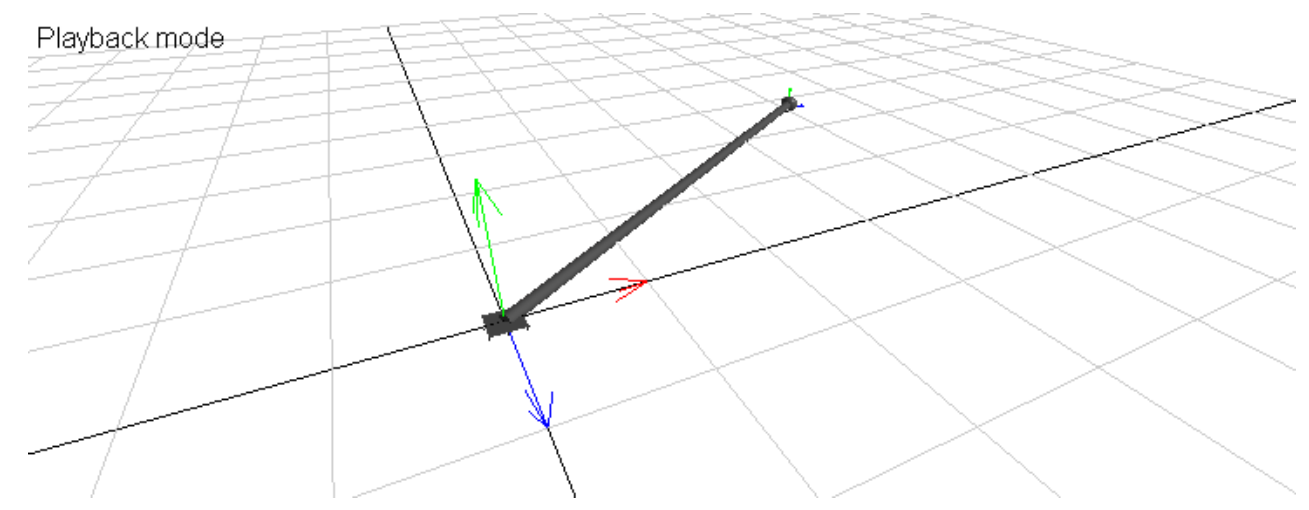

Fig. 7: A 3-D view of the Centrifuge model

Reference:

Halliday et al. "Fundamentals of Physics", 7th Edition. 111 River Street, NJ, 2005, John Wiley \& Sons, Inc. 